\title{
TRAITEMENT DES HELMINTHIASES ÉQUINES PAR LE PÉTROLE
}

\author{
Par L. FAURE
}

Nos équidés hébergent, dans leur tube digestif, de nombreux nématodes, responsables de troubles variés : amaigrissement, anémie, entérite parasitaire, cachexie vermineuse, phénomènes nerveux, etc... La pratique des autopsies confirme la fréquence des infestations massives et des associations parasitaires. En ce cas, il est difficile de préciser le rôle respectif des espèces ordinairement incriminées : oxyures, ascaris, sclérostomes, cylicostomes.

La découverte d'anthelminthiques appropriés a incité à de nombreuses recherches et de nouveaux produits (Didakol, Didakène, Vitan, Pyréthrine...) ont récemment enrichi notre vieille pharmacopée, à base d'arsenic, calomel, émétique, essence de térébenthine, thymol, ete... Irréguliers et inconstants sont cependant les résultats obtenus et les accidents causés par certains médicaments, anciens ou modernes, ont mème fait renoncer à leur emploi. En définitive, la valeur anthelminthique de la majorité apparait, d'une part, quelque peu surfaite, et ils ont, d'autre part, une action spécifique monovalente comme défaut principal.

La lutte contre ces agents pathogènes devient en campagne un grave problème, de nombreux chevaux et mulets de réquisition n'ayant jamais été traités à cet égard. Aussi, croyons-nous intéressant de signaler les avantages réels du «pétrole », anthelminthique polyvalent, fidèle, d'une innocuité absolue, d'un prix extrêmement modique, que l'on trouve partout. A ces divers titres, il pourra rendre de précieux services aux vétérinaires du front, des hôpitaux ou des dépôts qui sont plus ou moins désarmés contre ces affections et n'ont guère la possibilité ni les loisirs de s'astreindre à poser un diagnostic différentiel.

Boulay en préconisait l'utilisation il y a plus d'un demi-siècle. Les praticiens ne mirent aucun empressement à vanter l'efficacité d'un remède que leurs clients risquaient d'employer sans intervention du vétérinaire et ce fut, estimons-nous, la raison majeure

Annales de Pamastrologie, T, XVII, $\mathrm{x}^{\circ} 6,-1939-1940, \mathrm{p}, 590-592$. 
de son abandon. Délaissé à tort, il doit retrouver place dans notre arsenal thérapeutique.

Le mélange initialement essayé, pétrole et essence de térébenthine, était irritant pour le rein ; nous avons rapidement reconnu qu'administré seul, le pétrole donnait d'excellents résultats et ne présentait aucun danger. L'on ne peut escompter un succès complet qu'en soumettant au préalable l'animal à une diète hydrique rigoureuse de 24 heures au moins, 36 heures si possible, l'action toxique du pétrole, comme celle d'ailleurs de la majorité des vermifuges, étant grandement favorisée par la vacuité des réservoirs intestinaux.

Le pétrole doit être donné à la bouteille ou à l'aide de la seringue et des pinces labiales ; la dose globale $\left(0 \mathrm{~cm}^{3}, 5\right.$ à $0 \mathrm{~cm}^{3}, 7$ par kilogramme de poids vif) est avantageusement répartie en trois prises, administrées à jeûn, à 24 heures d'intervalle. L'introduction dans la cavité buccale d'un peu d'eau pure, à la suite du breuvage médicamenteux, assure sa déglutition totale. Les avantages du traitement fractionné sont: entretien de l'effet anthelminthique, meilleure répartition d'où action polyvalente plus sûre, expulsion plus rigoureuse, notamment des ascaris, imparfaitement obtenue avec une dose unique massive ; moindre risque d'irritation gastro-intestinale et suspension possible du traitement si l'animal parait incommodé à la suite d'une ou deux prises médicamenteuses.

Bien qu'il s'agisse d'une substance à la fois vermicide et vermifuge, l'adjonction d'huile comestible ou d'huile de ricin comme véhicule, agent de dilution et évacuant, est recommandable.

Notre expérimentation a principalement porté sur des chevaux barbes, d'un poids compris entre 300 et 360 kilogrammes. Après 36 heures de diète, nous donnions, à jeûn, une première dose de $60 \mathrm{gr}$. de pétrole en suspension dans $300 \mathrm{gr}$. d'huile d'arachides et une égale quantité d'eau; le même traitement était appliqué le lendemain; le jour suivant, pour la troisième et dernière prise, de l'huile de ricin était substituée à l'huile d'arachides.

La médication est toujours bien supportée, même par des sujets anémiés, débiles ; il n’y a, à notre connaissance, aucune contreindication. Après la troisième intervention, le malade rejette des excréments mous, puis diarrhéiques, exhalant une forte odeur sui generis. On y trouve, selon les cas, des représentants plus ou moins nombreux de la faune parasitaire. L'identification des parasites recueillis, maintes fois effectuée, confirme la présence d'ascaris, d'oxyures, de strongles (S. equinus, S. edentatus, S. vulgaris) et cylicostomes (C. catinatum, C. coronatum, C. tetracanthum); en 
principe, ascaris et oxyures sont expulsés morts ; il n'est pas rare, en revanche, de retrouver des strongles ténus encore vivants.

Chez un cheval français, de trait léger, atteint d'ascaridiase rebelle, nous avons porté à $240 \mathrm{gr}$. la dose totale de pétrole. L'animal bouda sur sa ration, présenta de l'inquiétude, de légers frissons, des coliques sourdes, mais aucun trouble grave; bientôt survint une diarrhée profuse où foisonnaient les ascaris : on put en compter plus de 1.100 dans les matières rejetées en une seule nuit.

L'expulsion massive des vers, l'absence d'œufs dans les excréments au cours des examens coprologiques pratiqués dans les semaines qui suivent, une reprise d'embonpoint rapide sont autant de preuves sérieuses de l'efficacité d'une médication qui, dans les circonstances actuelles, peut être, pour nos confrères, une arme précieuse.

\section{NOTES ET INFORMATIONS}

Localités nouvelles de Seine-et-Oise pour PhLebotomus perniciosus. - Cette espèce n'avait èté signalée jusqu'à ce jour en Seine-et-Oise que dans une seule localité : Brunoy (1). Deux nouveaux exemplaires en ont été recueillis pendant l'été de 1939 dans ce département, à Etampes et à Pontoise.

Un exemplaire femelle a èté récolté par l'un de nous (G. L.), à Etampes, le 30 août, à 6 heures du soir, donc en pleine lumière diurne et en plein air, au moment où il se préparait à piquer.

L'autre spécimen femelle a été capturé (A. R.) voltigeant sur des vitres à l'intérieur d'une maison, à 7 heures du soir, le 2 août 1939, à Pontoise.

Ces exemplaires, bíen caractéristiques de l'espèce, ont été déposés au Laboratoire de Parasitologie de la Faculté de Médecine de Paris.

G. Lavier et A. Ristorcell.t.

(1) Langeron (M.) et Nitzulescu (V.). - Révision des Phlébotomes de France. Ann. Parasit, X, 1932, p. 286.

Le Gérant: F. Amirault.

Imprimé par Imp: A. Coueslant (personnel intéressé)

à Cahors (France). -59.552 